\title{
Pregnancy-Induced Acute Intestinal Infarction in a Woman with Chronic Idiopathic Mesenteric Vein Thrombosis under Regular Anticoagulation Treatment
}

\author{
Chia-Meng Chan Wei-Lung Chen Jiann-Hwa Chen Yung-Lung Wu \\ Chien-Cheng Huang
}

Department of Emergency Medicine, Cathay General Hospital, and Fu Jen Catholic University School of Medicine, Taipei, Taiwan

\author{
Key Words \\ Intestinal infarction - Chronic idiopathic mesenteric vein \\ thrombosis · Pregnancy
}

\begin{abstract}
Objective: We present a case of acute intestinal infarction in a pregnant woman with chronic idiopathic mesenteric vein thrombosis (MVT) under regular anticoagulation treatment. Case Presentation and Intervention: The condition of the 26-year-old woman who was diagnosed with chronic idiopathic MVT after detailed investigation was stable after receiving regular anticoagulation with warfarin. One year later, she presented with a 7-day episode of intermittent epigastric pain. Acute intestinal infarction and concomitant 7-week pregnancy were diagnosed. To preserve her life, a dilation and curettage procedure and emergency laparotomy with bowel resection were performed. Ten days later, she was discharged, having made a good recovery. Conclusion: Although pregnancy was not the primary cause of chronic MVT, it did play a role in inducing the acute intestinal infarction. This case indicates that pregnant patients with known chronic idiopathic MVT should be counseled about the high risk of acute mesenteric thrombosis. This case also serves to remind physicians that there should always be a high level of suspicion of intestinal infarction in patients with an acute abdomen who are in a hypercoagulable state.
\end{abstract}

Copyright $\odot 2009$ S. Karger AG, Basel

\section{KARGER}

Fax +4161306 1234

E-Mail karger@karger.ch

www.karger.com
(C) 2009 S. Karger AG, Basel

$1011-7571 / 09 / 0185-0422 \$ 26.00 / 0$

Accessible online at:

www.karger.com/mpp

\section{Introduction}

Mesenteric vein thrombosis (MVT) is rare and difficult to diagnose due to its nonspecific presentation. If diagnosis or treatment were delayed, it can be fatal because intestinal infarction or variceal bleeding may occur [1-3]. Pregnancy complicating chronic idiopathic MVT has not been reported. We present a woman with chronic idiopathic MVT receiving regular anticoagulation for 1 year. However, intestinal infarction still occurred when she was 7 weeks pregnant.

\section{Case Report}

A 26-year-old woman was healthy and did not take any regular medication. One year prior to admission, portal vein thrombosis, superior MVT, splenomegaly and esophageal varices were found. No contributing cause could be identified after detailed examination including coagulation function. Warfarin was prescribed to treat and prevent further thrombosis. During a 1-year follow-up, her condition was stable without any abdominal complaints. The international normalized ratio was kept between 1.5 and 2.5.

However, she started to suffer from intermittent epigastric pain 7 days before the emergency department visit and was found to have been pregnant for 7 weeks prior to this episode. The pain had progressed to the entire abdomen with vomiting of bile 3 days prior to the visit; however, she was conscious with stable vital signs (blood pressure 102/86 mm Hg; hear rate 76/min; respira-

Chien-Cheng Huang, MD

Department of Emergency Medicine, Cathay General Hospital

280 Renai Rd. Sec. 4, Taipei 106 (Taiwan)

Tel. +8862 2708 2121, Fax +88622702 1428

E-Mail chienchenghuang@yahoo.com.tw 


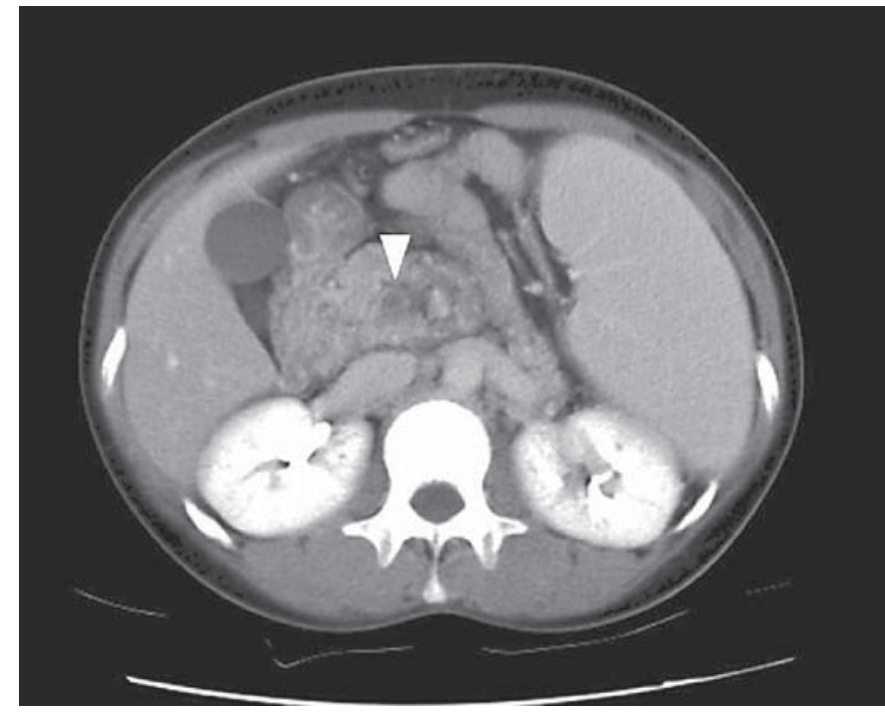

Fig. 1. Contrast-enhanced abdominal CT showed large collaterals in the porta hepatis, peripancreatic region, splenomegaly and nonvisualization of the superior mesenteric vein (arrowhead).

tory rate $17 / \mathrm{min}$; body temperature $36.6^{\circ} \mathrm{C}$ ). Physical examination revealed diffuse abdominal tenderness with muscle guarding. Routine laboratory data were within the normal range except for an elevated white blood cell count $(18,780 / \mathrm{ml})$, prolonged international normalized ratio (1.53) and elevated C-reactive protein $(1.945 \mathrm{mg} / \mathrm{dl})$. Plain film showed a localized ileus on the lower abdomen. Contrast-enhanced abdominal computed tomography (CT) showed MVT, splenomegaly and a thickened intestinal wall which suggested infarction (fig. 1). After discussion with the patient, emergency laparotomy was performed following dilation and curettage procedure. The operative finding was MVT and ischemic necrosis of the jejunum about $50 \mathrm{~cm}$ from the Treitz ligament. Resection of the infarcted bowel was done. The pathology showed transmural hemorrhagic necrosis. After 10 days of treatment, she was discharged with good recovery. Her condition was stable during a 1-year follow-up without anticoagulation treatment.

\section{Discussion}

It was the report by Warren and Eberhard [2] that helped to establish MVT as a distinct clinical entity. In the study by Rhee and Gloviczki [4], MVT comprised only $6.2 \%$ of all patients treated for mesenteric ischemia. Clinical symptoms of MVT are variable, nonspecific and difficult to differentiate from arterial occlusion. Patients may have a past medical or family history of thrombosis [1]. In most cases of acute MVT, the main symptom is abdominal pain lasting from several days to more than
3 months. Other symptoms are nausea/vomiting, fever and abdominal distention. Chronic MVT is usually asymptomatic due to the development of collateral vessels, but when it is combined with portal vein thrombosis, esophageal variceal bleeding may occur $[1,5]$. Our patient presented with a chronic course. Abdominal discomfort subsided after treatment with warfarin. No variceal bleeding occurred although esophageal varices had been found since the first diagnosis.

The laboratory findings of MVT are neither sensitive nor specific [1]. Diagnosis relies on CT and magnetic resonance imaging, combined with a high level of suspicion. Abdominal radiographs may demonstrate nonspecific ileus. Contrast-enhanced abdominal CT currently holds a sensitivity of $>90 \%$ and is the most common diagnostic test of choice. Magnetic resonance imaging offers no distinctive advantage over CT [1]. Angiographic findings are highly sensitive for vascular narrowing or stenosis when the ischemia is arterial in origin. However, the finding of veno-occlusive disease is less sensitive. In recent studies, angiography showed only 55\% sensitivity and has not been suggested as a primary diagnostic modality $[1,6]$. Duplex ultrasonography is also a viable option in diagnosing MVT but its role remains to be defined [1]. In our case, contrast CT was sufficient to reach a diagnosis.

Most patients have thrombotic tendencies or risk factors [1]. The predisposing factors are hypercoagulable states (protein $\mathrm{C}$ deficiency, protein $\mathrm{S}$ deficiency, antiphospholipid antibody, factor $\mathrm{V}$ Leiden mutation and antithrombin III deficiency), inflammatory state (appendicitis, diverticulitis, pancreatitis), portal hypertension, pregnancy, oral contraceptive use, abdominal injury and cancer $[1,3]$. In our patient, chronic MVT developed before her pregnancy and no risk factors could be identified after detailed investigation. Pregnancy seems to have had no direct relationship with chronic thrombosis, but it may have played an inducing role in the subsequent acute exacerbation.

Treatment of MVT depends on the stage of the disease [1]. Explorative laparotomy is indicated for patients with intestinal infarction as in our case. In the patients without bowel infarction or peritonitis, anticoagulation with heparin followed by warfarin is the mainstream therapy [1]. Other treatments such as peripheral or regional thrombolysis with or without surgical thrombectomy and a combination of surgical thrombectomy and regional heparinization have been reported [7-10]. Most researchers recommend maintaining anticoagulation therapy in order to prevent recurrent thrombosis, but the benefit is still criticized in some reports $[1,3,4,11,12]$. 
Our patient had received anticoagulation since her first diagnosis of MVT; however, acute exacerbation with intestinal infarction still developed. We cannot conclude that anticoagulation failed according to this single case report. But, it suggests that more aggressive prevention such as avoiding pregnancy or adding anti-platelet drug may be needed in this kind of patients with superimposed thrombotic risk.

The prognosis of MVT does not appear as ominous as that associated with arterial thrombosis [1], but its mortality remains as high as 30\% [2]. Our patient recovered very well after the operation. She did not receive further anticoagulation due to personal factors. Long-term follow-up will be needed.

\section{Conclusion}

This patient is the first case reported for acute intestinal infarction in pregnancy with chronic idiopathic MVT under anticoagulation treatment. The primary cause of chronic MVT was unknown and subsequent pregnancy might have played an inducing role for the intestinal infarction. This case indicates that pregnant patients with known chronic idiopathic MVT should be counseled about the high risk of acute mesenteric thrombosis. It also serves to remind physicians that there should always be a high level of suspicion of intestinal infarction in patients with an acute abdomen who are in a hypercoagulable state.

\section{References}

$>1$ Morasch MD, Ebaugh JL, Chiou AC, Matsumura JS, Pearce WH, Yao JS: Mesenteric venous thrombosis: a changing clinical entity. J Vasc Surg 2001;34:680-684.

$\checkmark 2$ Warren S, Eberhard TP: Mesenteric vein thrombosis. Surg Gynecol Obstet 1935;61: 102-121.

$>3$ Grendell JH, Ockner RK: Mesenteric venous thrombosis. Gastroenterology 1982;82:358372.

$\checkmark 4$ Rhee RY, Gloviczki P: Mesenteric venous thrombosis. Surg Clin North Am 1997;77: 327-338.

5 Goldstone J, Moore WS, Hall AD: Chronic occlusion of the superior mesenteric veins: report of a case. Am Surg 1970;36:235-237.
6 Eillis DJ, Brandt LJ: Mesenteric venous thrombosis. Gastroenterologist 1994;2:293298.

7 Demertzis S, Ringe B, Gulba D, Rosenthal H, Pichlmayr R: Treatment of portal vein thrombosis by thrombectomy and regional thrombolysis. Surgery 1994;115:389-393.

$>8$ Yankes JR, Uglietta JP, Grant J, Braun SD: Percutaneous transhepatic recanalization and thrombolysis of the superior mesenteric vein. AJR Am J Roentgenol 1988;151:289290.

$>9$ Rivitz SM, Geller SC, Hahn C, Waltman AC: Treatment of acute mesenteric venous thrombosis with transjugular intramesenteric urokinase infusion. J Vasc Interv Radiol 1995;6:219-228.
10 Olah A, Decurtins M, Largiader F: Die chirurgische Therapie der frischen Pfortaderthrombose. Helv Chir Acta 1990;57:5155.

11 Valla D, Denninger MH, Delvigne JM, Rueff B, Benhamou JP: Portal vein thrombosis with ruptured oesophageal varices as presenting manifestation of hereditary protein C deficiency. Gut 1988;29:856-859.

12 Brown KM, Kaplan MM, Donowitz M: Extrahepatic portal vein thrombosis: frequent recognition of associated diseases. J Clin Gastroenterol 1985;7:153-159. 